\title{
The hot air and cold facts of dietary fibre
}

\author{
Carla S Coffin MD FRCPC, Eldon A Shaffer MD FRCPC
}

America is a constipated nation.... If you pass small stools, you have to have large hospitals - Denis Burkitt

$\mathrm{D}_{\mathrm{t}}^{\mathrm{es}}$ enis Burkitt, a one-eyed Irish surgeon and physician (1911 to 1993), set his goal as a medical missionary in Africa. An astute clinician, he and his colleagues identified, in 1957, the lymphoma that bears his name. His next success in observing disease patterns came as the 'fibreman'. While in Uganda, he observed that Africans produced several times more feces than did westernized people. Further, the stool was more easily produced with minimal discomfort. He stated from his epidemiological studies:

"In Africa, treating people who live largely off the land on vegetables they grow, I hardly ever saw cases of many of the most common diseases in the United States and England - including coronary heart disease, adult-onset diabetes, varicose veins, obesity, diverticulitis, appendicitis, gallstones, dental cavities, hemorrhoids, hiatal hernias and constipation. Western diets are so low on bulk and so dense in calories, that our intestines just don't pass enough volume to remain healthy."

He believed that these western disorders had a single causative factor: deficiency of dietary fibre (1). He also hypothesized and instituted a worldwide crusade that fibre protected against colorectal cancer $(2,3)$. Such benefits of dietary fibre, a simplistic approach to a wide variety of diseases, were readily accepted by the medical community - often without question and often promulgated by the cereal and fibre manufactures. (Fibre consists of complex carbohydrates that are contained in plants that the body is unable to digest. Dietary fibre is classified as either soluble fibre or insoluble fibre, depending on its solubility in water and other factors. Soluble fibre binds bile and delays the time taken for digested food to move through the intestines. Insoluble fibre speeds up the process of food travelling through the intestines). How valid are the observations and beliefs of this medical icon today?

Epidemiological and animal studies have suggested that dietary fibre acts as a 'colonic broom', diluting fecal carcinogens and secondary bile acids (that may promote tumours by increasing colonic cell proliferation or by mutagenesis) and reducing intestinal transit time, hence limiting colonic exposure to carcinogens. From a different perspective, not espoused by Burkitt, the common unhealthy diet in the western world is characterized by high fat dairy products, sweets, refined grains, processed meat and lower intakes of whole grains and fibre $(4,5)$. All are now linked to hyperinsulinemia, which may act as a growth factor and tumour promoter.

A recent pooled analysis of 13 prospective cohort studies (6) found that dietary fibre was not associated with a reduced risk of colorectal cancer after adjusting for other dietary risk factors. The Cochrane collaboration (7) systematically reviewed five studies of over 4000 subjects for the effect of dietary fibre on the incidence or recurrence of colorectal adenomas and incidence of colorectal cancer over a two- to four-year period. The population included all subjects that had adenomatous polyps but no history of colorectal cancer or a documented 'clean colon' at baseline with follow-up colonoscopy. Study interventions included soluble and insoluble dietary fibre or a comprehensive dietary intervention with high fibre whole food sources. The combined data showed no outcome difference between the intervention and control groups in the number of subjects with at least one adenoma or a new diagnosis of colorectal cancer. The Cochrane reviewers (7) concluded that there was no evidence from randomized controlled trials to suggest that increased dietary fibre intake would reduce the incidence or recurrence of adenomatous polyps.

Widespread popular media advertisements have purported the benefits of soluble fibre in lowering the risk of atherosclerotic coronary artery disease, mainly by modifying the main coronary artery disease risk factors (ie, dyslipidemia, diabetes and obesity). As for diabetes, high fibre diets slow the postprandial rise in blood glucose and thus, improve glycemic control (8). In dyslipidemic patients, pundits have proposed that psyllium lowers serum cholesterol by binding bile acids in the intestinal lumen resulting in decreased absorption and increased fecal excretion. The ensuing bile acid depletion increases hepatic demand for the de novo synthesis of bile acids from cholesterol. Investigating this mechanism, Van Rosendaal et al (9) found that fibre administration had no effect and certainly did not lower serum cholesterol. Similarly, an earlier study (10) comparing the effect of wheat bran on serum cholesterol of hyperlipidemic and normolipidemic controls showed no change in total cholesterol or ratio of low density lipoprotein to high density lipoprotein cholesterol.

Division of Gastroenterology, Faculty of Medicine, University of Calgary, Calgary, Alberta

Correspondence: Dr Eldon A Shaffer, Division of Gastroenterology, Faculty of Medicine, University of Calgary, 3330 Hospital Drive South West,

Calgary, Alberta T2N 4N1. Telephone 403-210-9363, fax 403-210-9358, e-mail shaffer@ucalgary.ca

Received for publication July 28, 2005. Accepted August 2, 2005 
Another trial (11) of intensive dietary advice regarding fat, cereal fibre and fish intake on diet and mortality of men with a recent history of myocardial infarction did not find any substantial longterm benefit. The authors admitted to limitations of dietary data in the study (ie, only short-term period of advice and limited number of questions), but there was no evidence to guide decisions about value of dietary advice to increase fish or cereal fibre by people with coronary disease. We await the results of three Cochrane protocols undertaken to review the evidence of dietary fibre in fruits and vegetables, wholegrain cereals or high-fat, low fibre dietary intervention in the prevention of coronary heart disease (12-14). Any conclusions regarding the effectiveness of fibre for the prevention of heart disease appear premature.

Diverticular disease (DD), constipation and irritable bowel syndrome (IBS) are other common conditions that Trowell and Burkitt (15) attributed to a lack of dietary fibre. The pathogenesis of DD is multifactorial. Lack of dietary fibre contributes to slow transit, resulting in greater water absorption and subsequently smaller, harder stools that may lead to excessive colonic segmentation. Consumption of a high fibre diet helps lower colonic intraluminal pressure and minimizes bowel wall stress, possibly leading to a reduction in visceral pain due to wall tension (16).

In one of the first randomized, placebo-controlled trials of the role of bran in patients with DD (17), the authors concluded that dietary fibre supplements do nothing more than relieve constipation, and the impression that fibre helps DD is "simply a manifestation of western civilization's obsession with the need for frequent defecation". Recent systematic reviews $(18,19)$ of the role of dietary fibre and DD (both asymptomatic diverticulosis and symptomatic diverticulitis) conclude that most of the positive evidence of the effects of fibre supplementation in treating or preventing disease is from retrospective analyses with inherent limitations and high risk of bias. Although there is

\section{REFERENCES}

1. Burkitt DP. Are our commonest diseases preventable? Prev Med 1977;6:556-9.

2. Burkitt DP. Epidemiology of cancer of the colon and rectum. Cancer $1971 ; 28: 3-13$

3. Burkitt DP. An approach to the reduction of the most common western cancers. The failure of therapy to reduce disease. Arch Surgery 1991;126:345-7.

4. Bruce RW, Giacca A, Medline A. Possible mechanisms relating diet and risk of colon cancer. Cancer Epidemiol Biomarkers Prev 2000;9:1271-9.

5. Thomson CA, LeWinn K, Newton TR, Alberts DS, Martinez ME. Nutrition and diet in the development of gastrointestinal cancer. Curr Oncol Rep 2003;5:192-202.

6. Park Y, Hunter DJ, Spiegelman D, et al. Dietary fiber intake and risk of colorectal cancer: A pooled analysis of prospective cohort studies. JAMA 2005;294:2849-57.

7. Asano T, McLeod RS. Dietary fibre for the prevention of colorectal adenomas and carcinomas. Cochrane Database Syst Rev 2002; (2):CD003430.

8. Tuomilehto J, Lindstrom J, Eriksson JG, et al. Prevention of type 2 diabetes mellitus by changes in lifestyle among subjects with impaired glucose tolerance. N Engl J Med 2001;344:1343-50.

9. Van Rosendaal GM, Shaffer EA, Edwards AL, Brant R. Effect of time of administration on cholesterol-lowering by psyllium: A randomized cross-over study in normocholesterolemic or slightly hypercholesterolemic subjects. Nutr J 2004;3:17.

10. Jenkins DJ, Kendall CW, Axelsen M, Augustin LS, Vuksan V. Viscous and nonviscous fibres, nonabsorbable and low glycemic index carbohydrates, blood lipids and coronary heart disease. Curr Opin Lipidol 2000;11:49-56. strong evidence that dietary fibre, especially insoluble fibre in fruits and vegetables, decreases the risk of DD, other lifestyle factors such as lower red meat and fat consumption and physical exercise play a role in bowel function.

Increased dietary fibre as a bulking agent for management of constipation is often recommended for patients with IBS, even though these patients do not report less dietary fibre intake than control subjects (20). In practice, many patients with IBS complain of bloating with higher doses of natural fibre, likely due to bacterial fermentation producing shortchain fatty acids, increasing colonic gas and distension, and hence, aggravating IBS symptoms.

Systematic reviews have shown that the treatment of IBS patients with fibre is controversial. One recent meta-analysis of 17 randomized controlled trials (20) quantified the effectiveness of different types of fibre. The reviewers found that fibre was only marginally effective in terms of global symptom improvement or constipation and there was no effect in IBS related abdominal pain. Fibre has a role in treating constipation but its value for IBS, pain and diarrhea is controversial. Any effectivenss of fibre in the long-term management of IBS remains questionable. Clinically, bran is no better than placebo in the relief of the overall symptoms of IBS, and is possibly worse than a normal diet for some symptoms.

Fibre is therefore not a panacea for all ills as Burkitt suggested (1) over four decades ago. Although most gastroenterologists advise a high fibre diet, overall evidence suggests that a more critical look at the virtues of fibre is needed. Individuals who consume more fibre might have other healthy lifestyle attributes including smoking less, exercising more and consuming more fruits and vegetables, resulting in a halo effect for the benefits of fibre. Current evidence does not justify routine recommendations of fibre supplementation. Fibre may appear in decline as a factor in a multitude of diseases, but do not count it out yet.

11. Ness AR, Hughes J, Elwood PC, Whitley E, Smith GD, Burr ML. The long-term effect of dietary advice in men with coronary disease: Followup of the Diet and Reinfarction trial (DART). Eur J Clin Nutr 2002;56:512-8.

12. Brunner EJ, Thorogood M. Dietary interventions for reducing cardiovascular risk. Cochrane Database Syst Rev 2005;(3).

13. Kelly SAM, Brynes A, Frost G, Lang R, Whittaker V, Summerbell CD. Wholegrain cereals for coronary heart disease. Cochrane Database Syst $\operatorname{Rev} 2005 ;(3)$

14. Ness A, Hooper L, Egger M, Powles JW, Davey-Smith G. Fruits and vegetables for cardiovascular disease. Cochrane Database Syst Rev 2005; (3)

15. Trowell HC, Burkitt DP. The development of the concept of dietary fibre. Mol Aspects Med 1987;9:7-15.

16. Simmang CL, Shires GT. Diverticular disease of the colon. In: M Feldman, LS Friedman, MH Sleisenger, eds. Sleisenger and Fordtran's Gastrointestinal and Liver Disease. Pathophysiology/Diagnosis/ Management, 7th edn. Philadelphia: Saunders. 2002:2100-11.

17. Ornstein MH, Littlewood ER, Baird IM, Fowler J, North WR, Cox AG Are fibre supplements really necessary in diverticular disease of the colon? A controlled clinical trial. Br Med J (Clin Res Ed) 1981;282:1353-6

18. Aldoori W, Ryan-Harshman M. Preventing diverticular disease. Review of recent evidence on high-fibre diets. Can Fam Physician 2002;48:1632-7.

19 Murray CD, Emmanuel AV. Medical management of diverticular disease. Best Pract Res Clin Gastroenterol 2002;16:611-20.

20. Bijkerk CJ, Muris JW, Knottnerus JA, Hoes AW, de Wit NJ. Systematic review: The role of different types of fibre in the treatment of irritable bowel syndrome. Aliment Pharmacol Ther 2004;19:245-51. 


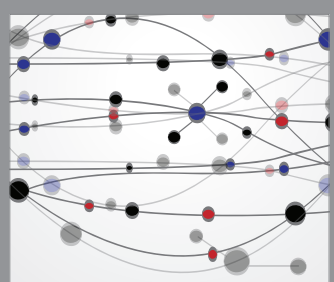

The Scientific World Journal
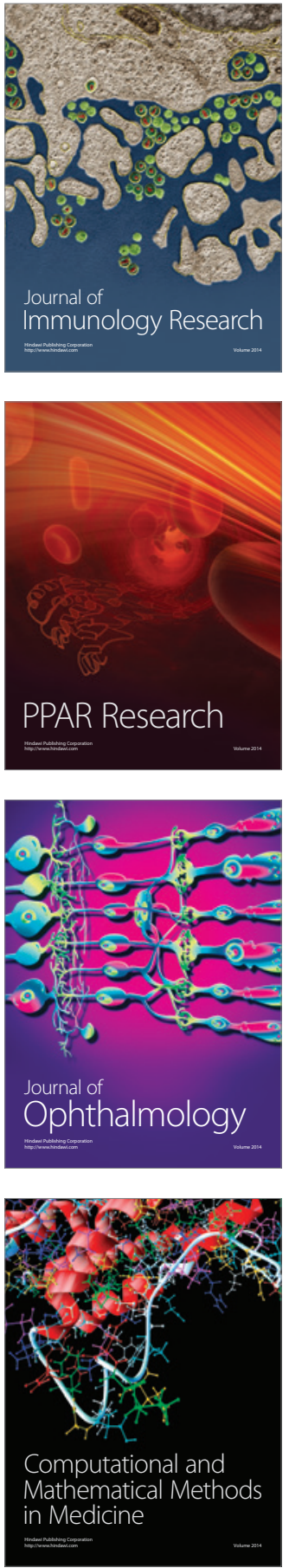

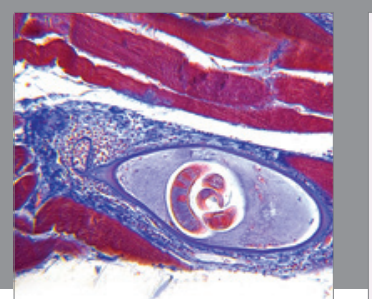

Gastroenterology Research and Practice

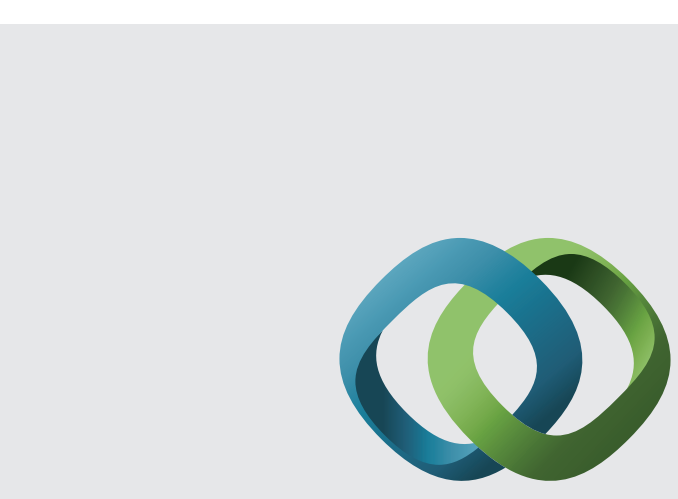

\section{Hindawi}

Submit your manuscripts at

http://www.hindawi.com
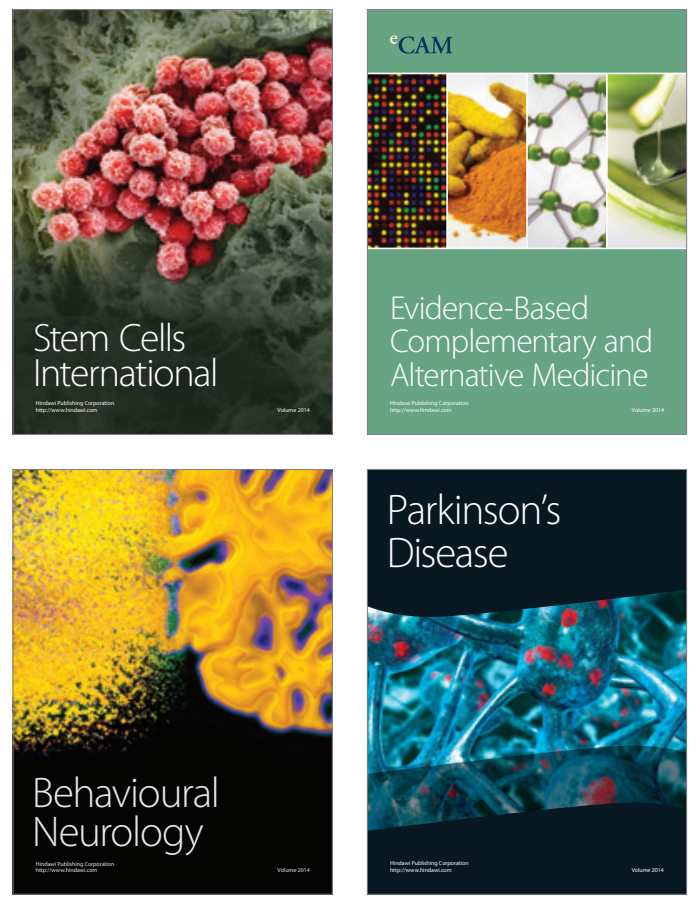
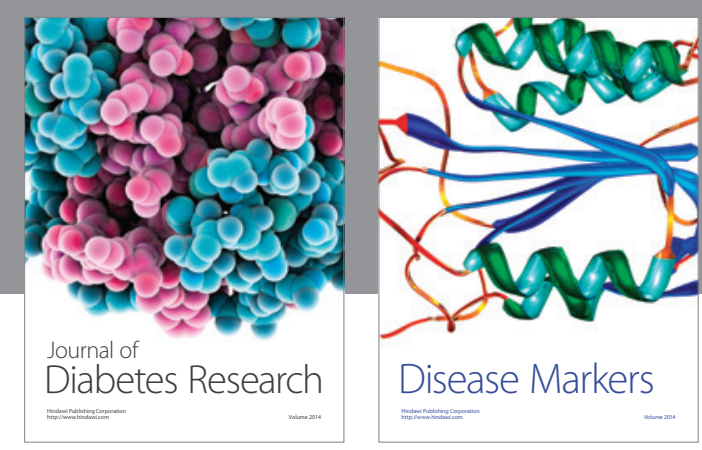

Disease Markers
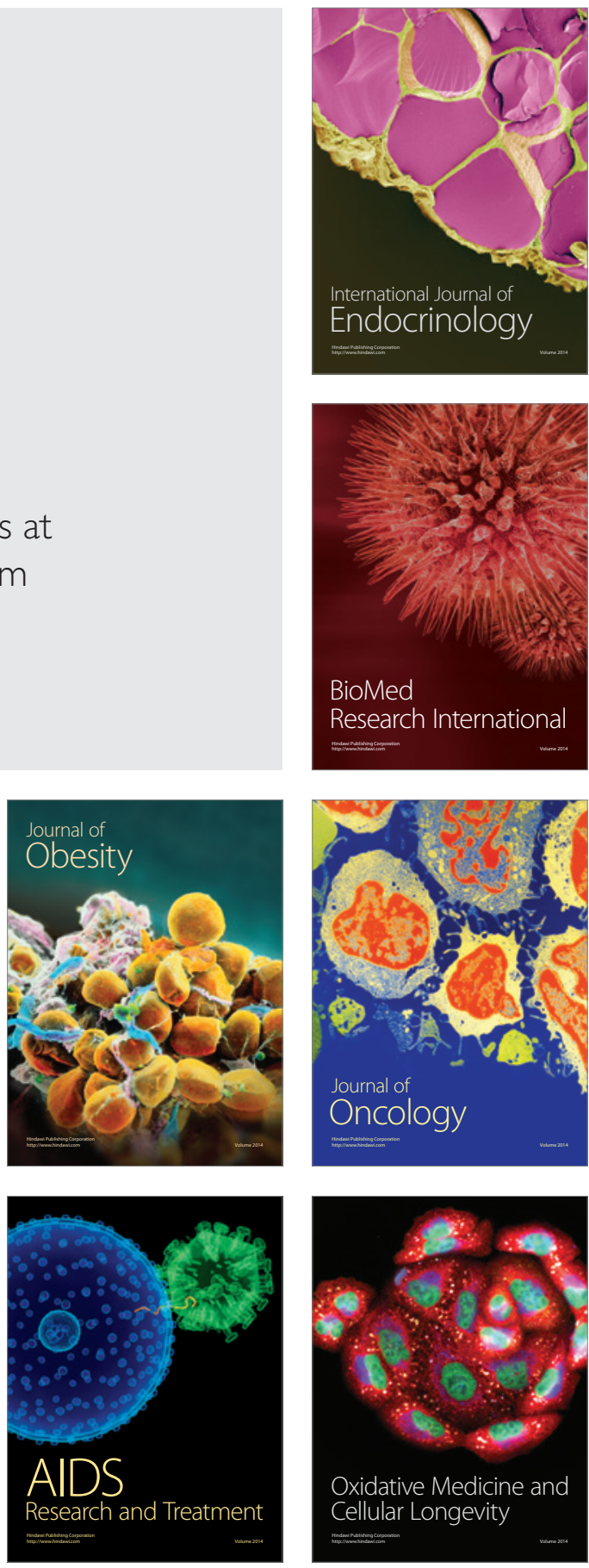